NBER WORKING PAPER SERIES

CONSENSUS AND UNCERTAINTY IN ECONOMIC PREDICTION

Victor Zarnowitz

Louis A. Lambros

Working Paper No. 1171

NATIONAL BUREAU OF ECONOMIC RESEARCH

1050 Massachusetts Avenue

Cambridge MA 02138

July 1983

Financial support from the National Science Foundation and aid of the Graduate School of Business of the University of Chicago are gratefully acknowledged. The research reported here is part of the NBER's research program in Economic Fluctuations. Any opinions expressed are those of the authors and not those of the National Bureau of Economic Research. 
NBER Working Paper 非1171

July 1983

\title{
CONSENSUS AND UNCERTAINTY IN BCONOMIC PREDICTION
}

\begin{abstract}
The usual practice in economic forecasting is to report point predictions without specifying the attached probabilities. Periodic surveys of such forecasts produce group averages, which are taken to indicate the "consensus" of experts. Measures of the dispersion of individual forecasts around these averages are interpreted as indicating "uncertainty."

However, consensus is best defined as the degree of agreement among the corresponding point predictions reported by different forecasters, while uncertainty is properly understood as referring to the diffuseness of the distributions of probabilities that individual forecasters attach to the different possible values of an economic variable. The NBER-ASA quarterly economic outlook surveys provide unique information on probabilistic forecast distributions reported by a large number of individuals for changes in GNP and the implicit price deflator in 1969-81. These data permit comparisons of related point and probability forecasts from the same sources.

The matched mean point forecasts and mean probability forecasts are found to agree closely. Standard deviations of point forecasts are generally smaller than the mean standard deviations of the predictive probability distributions for the same targets. Thus the former tend to understate uncertainty as measured by the latter. This is so particularly for short horizons.

Victor Zarnowitz

and

Louis Lambros

University of Chicago

Graduate School of Business

1101 East 58th Street

Chicago, Illinois 60637
\end{abstract}




\section{Introducing the Problems and Data}

Although all forecasts are by their very nature probabilistic statements, most economic predictions quote but a single value to be assumed by a certain variable, without specifying the attached probabilities. often many such point forecasts are available for a given target variable from a business outlook survey. If they show a high degree of agreement, does this indicate that the forecasters confidently expect the outcome they commonly predict to come true? More generally, does the dispersion of the point forecasts reflect their authors' uncertainty (i.e., their relative lack of confidence)? This paper will deal with these and other related questions, drawing on a set of data which is very rare in economics in that it includes related point and probabilistic forecasts from the same sources.

\section{Consensus}

Averages from economic outlook surveys are frequently called "consensus" forecasts or treated as such. The term has entered the popular discourse without having been defined in a generally accepted way. But it is clear that the degree to which a survey average is representative of the collected individual predictions can vary greatly depending on the nature of the underlying distribution. There may be no meaningful consensus if the distribution of the point forecasts in question is highly diffuse or multimodal; on the other hand, a consensus would be strongly in evidence for any unimodal, symmetrical, and sufficiently tight distribution (cf. Schnader and Stekler, 1979).

The point forecasts for some target variable $y_{t+j}$ obtained from a survey taken in period $t$ could be distributed as shown by the histograms in the left-hand panel of Figure 1. The smoothed frequency curves represent the corresponding theoretical distributions. In such cases the existence of a 
$\mathrm{n}$

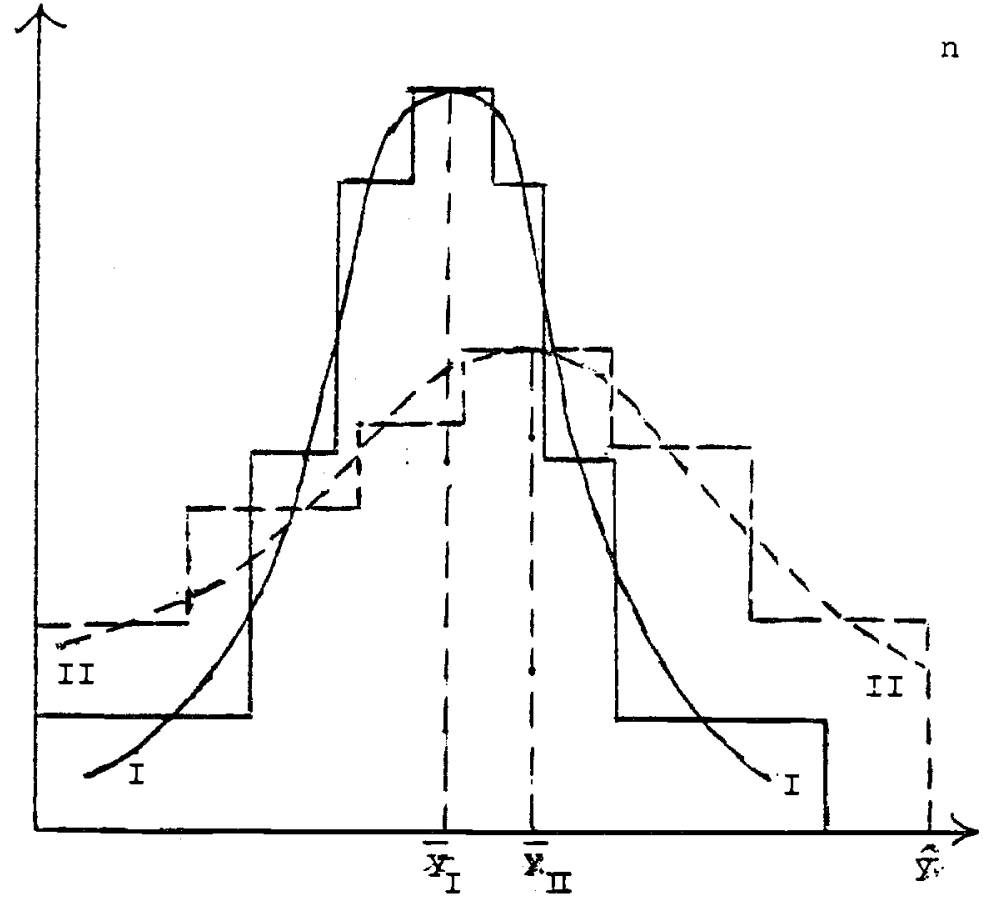

I: strong consensus; II: weak consensus

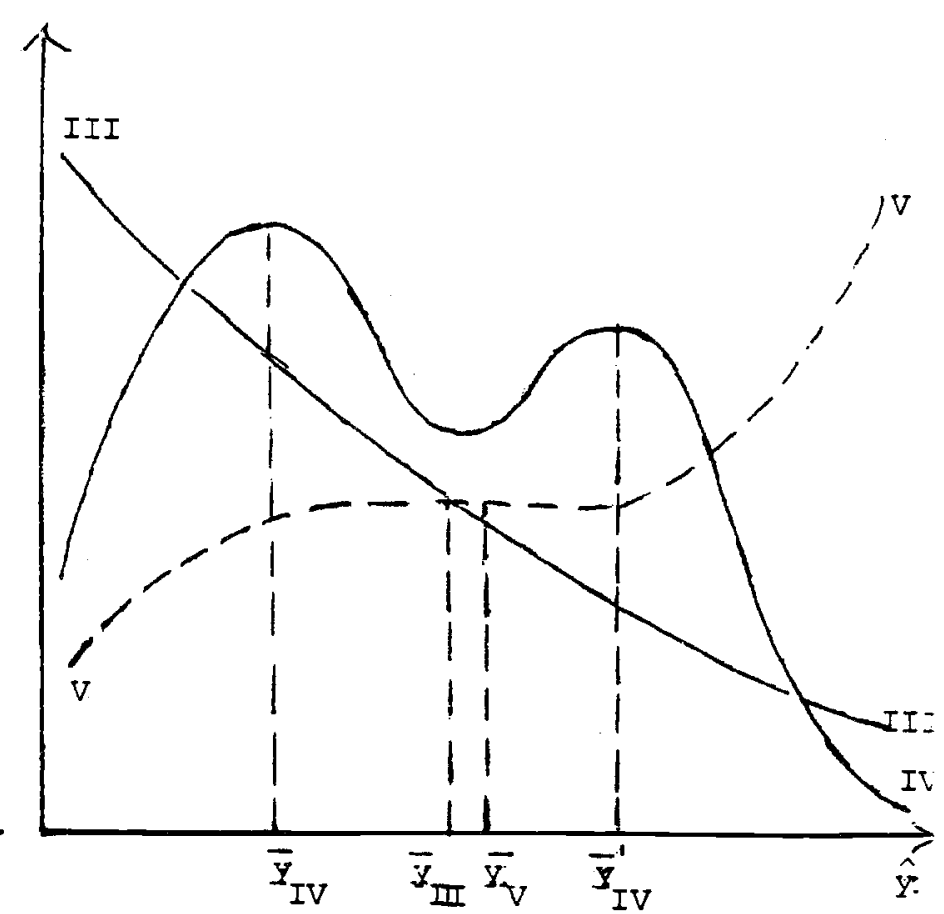

III, IV, V: no consensus?

LEGEND: I-V are smoothed frequency curves showing the frequencies $(n)$ of the point forecasts (i). These are theoretical distributions; the histograms illustrate the data corresponding to $I$ and $I I$. In cases $I$ and $I I$, average values such as $\bar{Y}_{I}$ and $\overline{\bar{Y}}$. may serve as consersus forecasts; in cases III, IV, and V no such representative values are available (see $\overline{\bar{x}}_{\text {III }}-\bar{Y}_{V}$ ). 
consensus is not in doubt, though the degree of consensus varies (it is clearly much higher for I than for II). In the right-hand panel, the distributions (shown for simplicity in smoothed form only) are strongly skewed or bimodal so that no well-defined consensus may exist.

Expectations or forecasts for the same aggregate variables are likely to draw upon common, publicly available information and widely recognized techniques and relationships (models). Also, people interact and influence each other directly or indirectly, through informal exchanges, opinion polls, media dissemination of public predictions, and market arrangements for the sale of expert advice. The lack of independence would tend to produce clustering, i.e., tightening of the representative distribution, of the point forecasts. This suggests an influential role for the prevalent "consensus" prediction from which many risk-averting forecasters may not wish to deviate much at any time.

For all the above reasons, positive correlations can be expected to prevail among the corresponding expert forecasts and agent expectations. Yet genuine predictions do not simply mimic one another but contain some independent information, so their collinearity will be limited and combining them should result in some net gains in accuracy. Indeed, simple averages of forecasts from successive business outlook surveys have proved to be more accurate over time than most of the corresponding forecast sets of the individual participants. 1

${ }^{1}$ See Zarnowitz, 1967, pp. 123-126, and 1983a. Methods to choose a diversified "portfolio" of forecasts and weights that reduce the variance of the resulting composite have been proposed in the literature, and some success in their application was reported (Bates and Granger, 1969; Newbold and Granger, 1974). On the conditions under which unweighted aggregate predictions are optimal or nearly optimal, see Einhorn and Hogarth, 1975; also, Hogarth, 1978 . 
An important aspect of the consensus is the dispersion of a sample of point forecasts, which can be measured simply by standard deviation. If the cross-sectional distributions of forecasts come in a variety of shapes illustrated in Figure 1, however, other aspects such as skewness and modality or kurtosis will also require attention.

\section{Uncertainty}

Recent studies have used the variance of predicted prices changes across survey respondents as a positive measure of "inflation uncertainty."2 To generalize, high (low) dispersion of point forecasts is thus interpreted as being indicative of high (low) uncertainty. Thus "uncertainty" here is simply an inverse of what was labeled "consensus" in the previous section.

It is important to recognize that this approach certainly does not involve any direct measurement of uncertainty in the usual sense of that term. The latter is a function of the distribution of the probabilities that a forecaster attaches to the different possible outcomes (values) of the predicted event (variable). The tighter this distribution, the lower is the associated uncertainty. Hence some knowledge of the probabilities involved would seem necessary for any outside assessment of this aspect of the situation. Inferences from point forecasts are altogether a different matter.

Suppose that the standard deviation of a set of corresponding predictions by different individuals is taken to indicate uncertainty. The underlying assumption is that this interpersonal dispersion measure is an acceptable proxy for the dispersion of intrapersonal predictive probabilities or beliefs held by the same individuals. The validity of this assumption can by no means be taken for granted.

${ }^{2}$ For references and results, see Makin, 1982. 
The point forecasts reported by the individuals A, B, C, ... may be viewed as the expected values of their respective probability distributions. The degree of consensus among the survey respondents is said to be "high" when their point predictions are clustered, "low" when they are widely dispersed. The degree of uncertainty is said to be "high" when the predictive probability distributions of $A, B, C, \ldots$ are diffuse, "low" when they are tight.

As illustrated in Figure 2, high consensus may be associated with either low or high uncertainty (compare panels a and b). Similarly, low consensus may be associated with either low or high uncertainty (panels $c$ and d). These graphs are believed to be self-explanatory, but it should be noted that they are of necessity starkly oversimplified. But the schematic diagrams suffice to support our main argument concerning the important distinction between consensus and uncertainty.

Recent literature has found both theoretical and empirical reasons to stress the role of uncertainty with regard to inflation. ${ }^{3}$ Uncertainty about real growth prospects received little attention but may deserve much more. The effects of changes in the "confidence" of consumers, investors, and businessmen are often emphasized, but these changes themselves and their determinants are extremely difficult to measure and analyze. There is little firm knowledge in this area, a major reason for this being the lack of data that are directly related to uncertainty as defined above.

Data

In the sphere of economics, point predictions dominate and reported probabilistic distribution predictions are regrettably rare. Business outlook

${ }^{3}$ Cukierman and Wachtel, 1979; Mullineaux, 1980; Levi and Makin, 1980; Makin, 1982. 

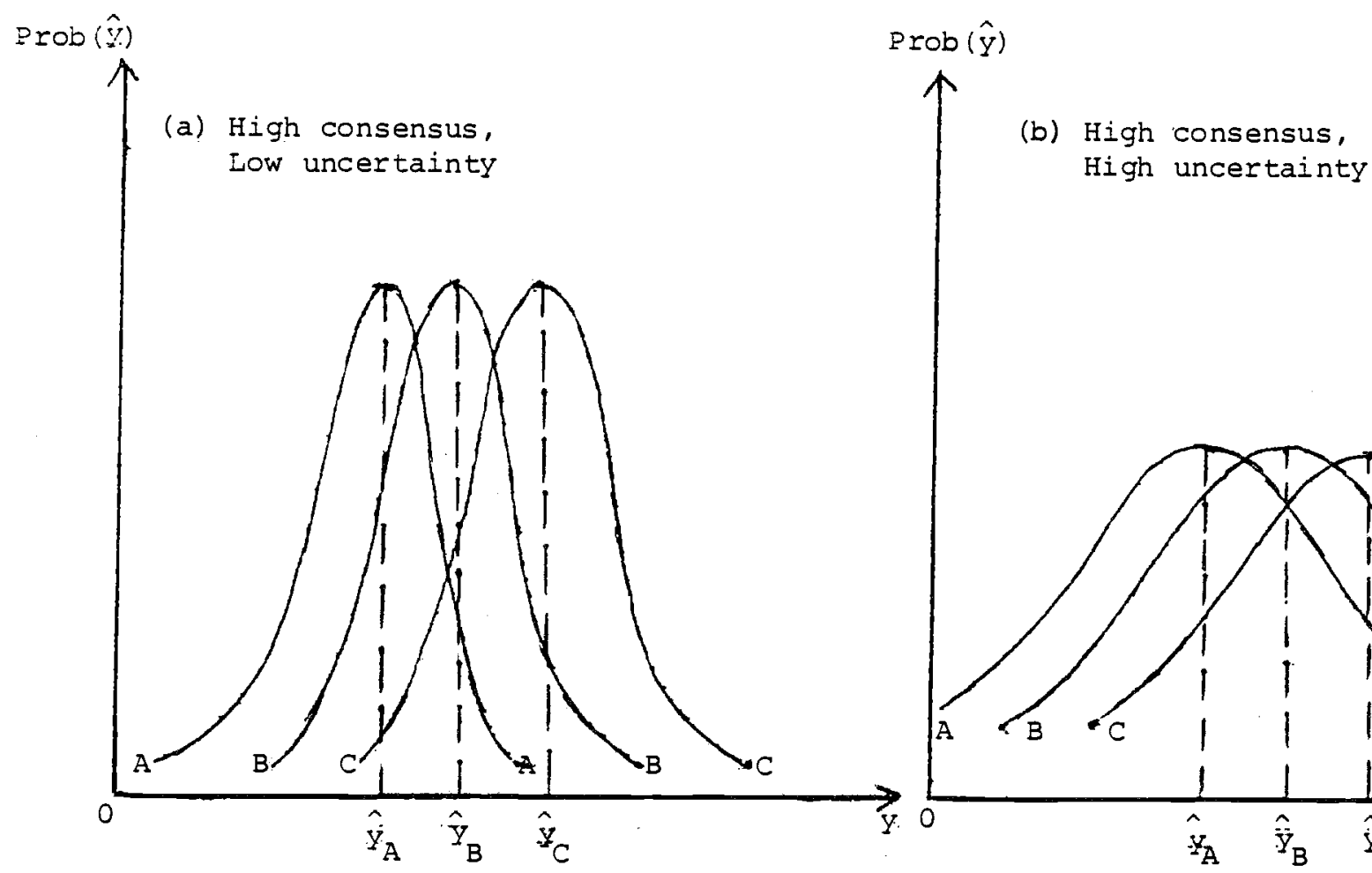

Prob $(\hat{y})$

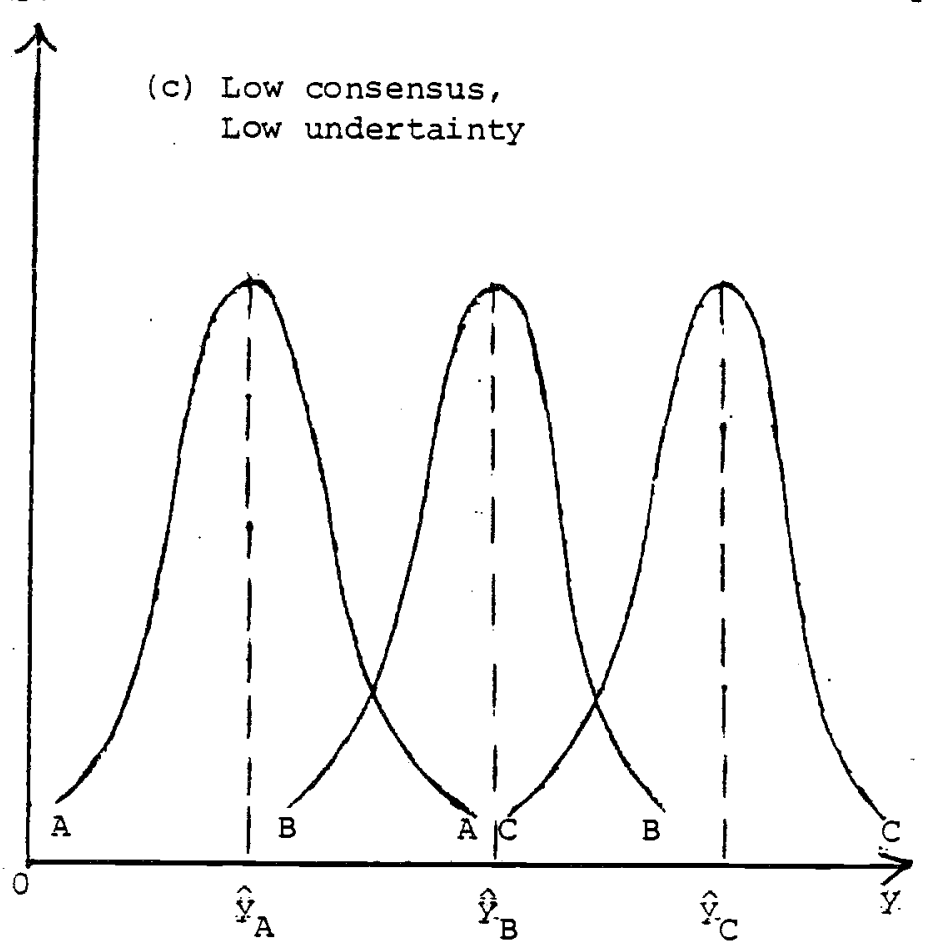

$\operatorname{Prob}(\hat{\mathrm{Y}})$
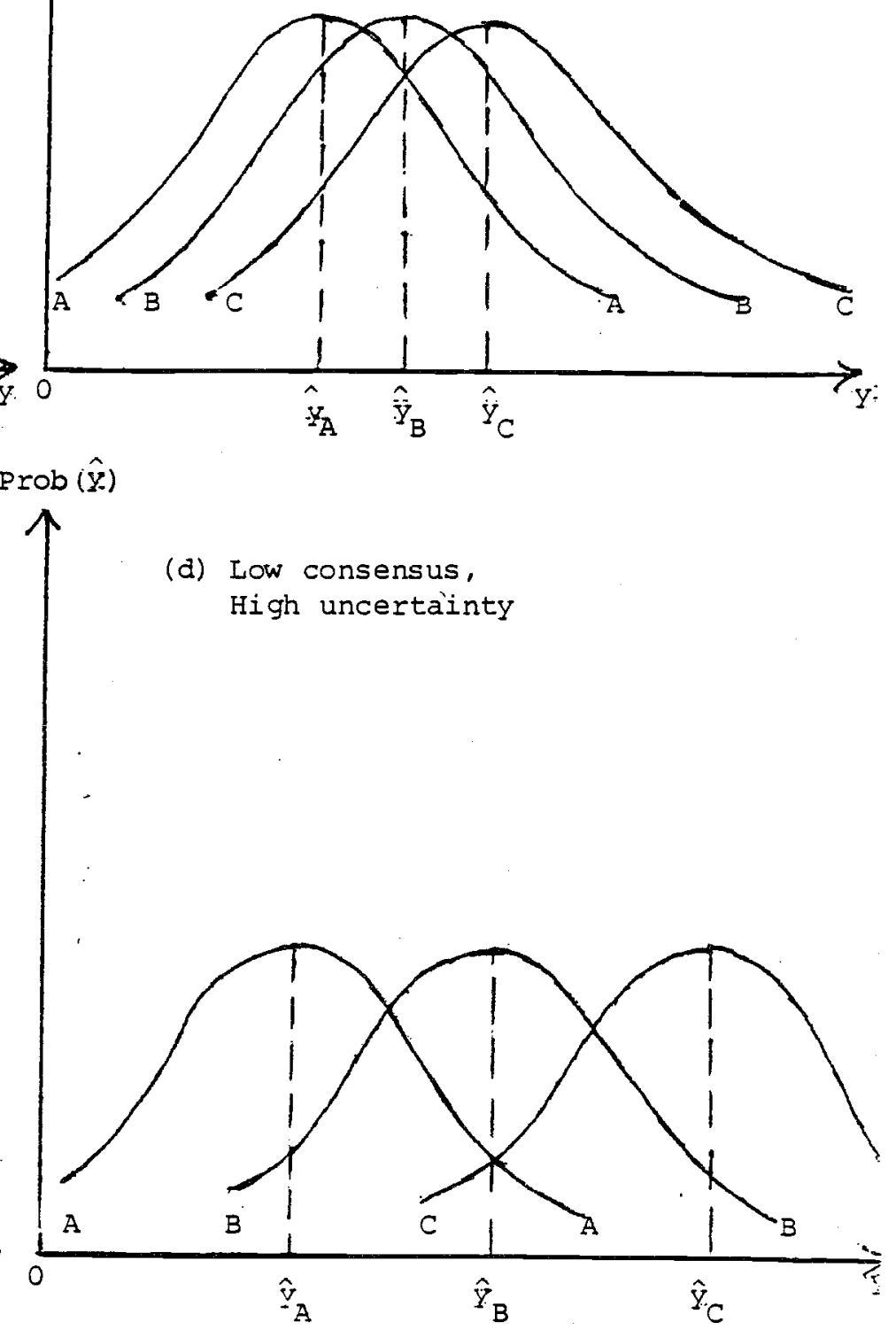

LEGEND: Curves $A, B$, and $C$ represent the probability distributions of alternative forecasts from sources A, B, and C, respectively. The probabilities Prob $(\hat{y})$ are measured vertically, the different values of the predicted variable (Y) are measured horizontally. $\hat{y}_{i}(i=A, B, C, \ldots)$ are the point forecasts. 
surveys generally do not collect information on the uncertainty that a participant connects with his or her forecast or expectation. The materials used in this study came from a survey which is to our knowledge unique in regularly eliciting some replies on this point.

The survey has been conducted quarterly since 1968 by the National Bureau of Economic Research (NBER) and the Business and Economic statistics Section of the American Statistical Association (ASA). The survey questionnaire, mailed by ASA, reaches a broadly based and diversified group of persons who are professionally engaged in the analysis of current and prospective business conditions. A report on each survey is prepared by NBER, and the historical results from the surveys have been used in basic studies by NBER authors and others. 4

The ASA-NBER survey members report their point predictions for more than ten important macroeconomic variables including the gross national product in current dollars and constant dollars and the GNP implicit price deflator (GNP, RGNP, and IPD, respectively). These forecasts refer to the current and the next four quarters. In addition, the respondents are asked to fill in the probabilities they attach to a list of possible year-to-year percentage changes in IPD and GNP (through mid-1981) or RGNP (thereafter). The probabilities are specified in percentage form, to add up to 100 in each case. Another regular question concerns the probabilities of a downturn in real GNP in each of the quarters covered.

This study covers 80 individuals who participated in at least 12 of the 51 quarterly ASA-NBER surveys taken during the period 1968:4-1981:2. The total number covered was 192, but this inciudes many sporadic respondents who could

${ }^{4}$ see Zarnowitz, $1982 \mathrm{~b}$ and 1983, and references therein for a description of the contents of the ASA-NBER surveys. 
not be individually evaluated because of paucity of data. The performance of these infrequent respondents was on the whole less satisfactory than that of the "regulars." Concentration on the latter also improves the quality of the data by reducing the variation of the coverage over time. 5

The actual and predicted values are expressed in terms of percentage changes, at unit period (quarterly or annual) rates. The actual values used to compute the forecast errors are the last estimates available prior to the benchmark revisions of January 1976 and December 1980.6

The sequences of quarters covered by the point forecasts overlap the years covered by the probabilistic forecasts, so that we are able to construct time series of summary measures for the two sets which are strictly comparable in coverage. That is, the paired series refer to the forecasts by the same individuals for the same target variables and periods. This analysis, presented in the immediately following section, addresses directly the questions raised before and forms the core of this paper.

\section{Time Series of Probabilistic Distribution Forecasts}

\section{Samples and Measures}

The NBER-ASA survey questionnaires include the question "Please indicate what probabilities you would attach to the following possible percentage changes from 19.. to 19.. in current dollar GNP and the implicit price deflator (annual figures) . . " followed by lists of one-percent intervals with blank

${ }^{5}$ Each of the 51 surveys still has an adequate coverage: on the average 41 participants, with a standard deviation of 10 , maximum of 60 , and minimum of 21. Each of the 80 individuals also had an adequate exposure: the range is 12 to 34 surveys, with a mean of 23 and a standard deviation of 8 .

${ }^{6}$ On the advantages and disadvantages of different measures of forecasts and errors, and on the role of revisions, see zarnowitz 1967, 1979, and 1982a; Cole, 1969; and McNees, 1973. 
spaces to write the numbers in. The replies specify the chances in 100 that the forecasters associate with the changes falling in the selected intervals. For example, an individual might enter the figures, $15,35,40$, and 10 in lines labeled +19.0 to $+11.9,+10.0$ to $+10.9,+9.0$ to +9.9 , and +8.0 to 8.9 percent, respectively, for the expected distribution of GNP changes in 1979-80.

Although the data concern annual changes from year $t-1$ to year $t$, they come from quarterly surveys extending over the two years, so that the predictions vary considerably in length. The four consecutive surveys from the last quarter of year $t-1$ through the third quarter of year $t$ provide the bulk of the information. The distances between the dates of these surveys and the end of the target year are approximately $4-1 / 2,3-1 / 2,2-1 / 2$, and $1-1 / 2$ quarters. Proceeding in the reverse order from the shortest to the longest forecasts, we shall refer to these categories simply as horizons 1, . . ., 4.

For the years 1969-81, the total number of the reported probabilitistic forecast distributions (pb) exceeded 4,600, about evenly divided between GNP and IPD. This "all respondents" sample was subjected to some analysis, but our main concern is with a smaller sample comprising only those "regulars" who participated in at least 12 of the 51 surveys covered. Earlier analysis of data from the same surveys, which had to impose some minimum-response restriction on the individual sets of point forecasts (pt) under study, used the "12 or more" rule to good advantage, and the approach is followed here in the interest of consistency and comparability.

We have screened the data from the completed questionnaire forms available in the NBER files so as to (1) strictly match the pb and pt forecasts made by the same persons for the same targets, and (2) eliminate unusable replies and obvious errors. This resulted in a collection of 3,378 individual pb distributions, with a matching number of corresponding pt predictions. The coverage 
characteristics of these samples are described by the accompanying tabulation.

\begin{tabular}{ccccc}
\hline \hline $\begin{array}{c}\text { Horizon } \\
\text { (quarters) }\end{array}$ & $\begin{array}{c}\text { Period covered } \\
\text { (number of years) }\end{array}$ & $\begin{array}{c}\text { Number (Percent) } \\
\text { GNP }\end{array}$ & $\begin{array}{c}\text { of Forecasts } \\
\text { IPD }\end{array}$ \\
1 & $\begin{array}{c}1969-71,73-78,80(10) \\
2 \\
3\end{array}$ & $1969-78,80-81(12)$ & $425(19.4)$ & $330(19.4)$ \\
4 & $1969-81(13)$ & $453(27.1)$ & $458(26.9)$ \\
Total & $1969-74,76-80(11)$ & $467(27.9)$ & $485(28.4)$ \\
\hline \hline
\end{tabular}

This collection does not include the $\mathrm{pb}$ predictions issued in the second and third quarter of year $t-1$ and in the fourth quarter of year $t$. The former cannot be matched with pt forecasts and the latter are extremely short, since by $Q 4$ of $t$ most of the target year is already over and covered by at least some preliminary "actuals." Moreover, these replies for horizons "6", "5" and "O" are available in one or two surveys only."

Summary statistics such as the mean, standard deviation, skewness, and kurtosis were calculated for each of the accepted individual probability distributions. Uniform distribution within each of the selected intervals was assumed. Thus the $\mathrm{k}^{\text {th }}$ order moment about zero of the distribution is computed by numerical integration as

7 The years covered are 1974, 1980, and 1981, and only 136 (about half) of these pb distributions have pt counterparts. In addition, careful editing led to the elimination of 210 faulty or unusable replies ( 120 for GNP, 90 for IPD) associated with the following reasons: degenerate distributions with single "100" entries, 116; cases in which the probabilities do not add up to 1.00, 47; mistaken applications to real rather than nominal GNP, 47 . 


$$
\mu_{k}^{0}=\sum_{i} p_{i}\left(\frac{u_{i}^{k+1}}{k+1}-\frac{e_{i}^{k}+1}{k+1}\right)
$$

where $p_{i}$ is the probability assigned to the $i^{\text {th }}$ interval $\left(\sum_{i} p_{i}=1\right)$, and $\ell_{i}$ and $u_{i}$ are the lower and upper limits of the $i^{\text {th }}$ interval, respectively. Since unit intervals are used, the mean $(k=1)$ reduces to $\sum_{i} p_{i}\left(\frac{l_{i}+u_{i}}{2}\right)$. The mean forecast implicit in the $j^{\text {th }}$ respondent's probability distribution for horizon $h$ and year $t$ will be denoted as $\phi_{\text {jht }}$ (All this applies, of course, to GNP and IPD forecasts alike.)

For each $\phi_{j h t}$ there is a matching point forecast $f_{j h t}$. The latter numbers are computed from corresponding estimates and predictions of quarterly levels. For example, in the fourth quarter of year $t_{04}$, a respondent would use data on the "actual" values of GNP in the preceeding quarters (... $\mathrm{A}_{02}, \mathrm{~A}_{03}$ ) and make predictions through the end of the year $t+1\left(P_{04}, P_{11}, \ldots, P_{14}\right)$. Accordingly, the annual percent change forecast for any $j$ and $t$ and for $\mathrm{h}=4 \mathrm{is}$

$$
f_{4}=\left(\frac{P_{11}+P_{12}+P_{13}+P_{14}}{A_{01}+A_{02}+A_{03}+P_{04}}-1\right) 100 .
$$

Similarly, $f_{3}$ made in the first quarter of the year $t+1$ would equal the ratio $100\left(\sum_{j=1}^{4} P_{i j} / L_{j=1}^{4} A_{0 j}-1\right)$, where the $P^{\prime} s$ and $A^{\prime} ' s$ are the new quarterly level predictions and estimated realizations, respectively (note that $P_{04}$ is now replaced by $\left.A_{04}\right)$. Still more recent predictions and estimates would be available for $f_{2}$ (including $A_{11}$, instead of $P_{11}$ ) and $f_{1}$ (including also $\mathrm{A}_{12}$ instead of $\mathrm{P}_{12}$ ).

The individual $\phi$ and $f$ predictions are used next to construct annual time series of group averages. Thus, the means of the individual probability distributions are averaged across all members of the "12 or more" sample participating in the given survey as in 


$$
\frac{1}{n} \sum_{j}^{n} \phi_{j h t}=\Phi_{h t} .
$$

The matching point forecasts are similarly averaged over the same individuals according to $\cos$

$$
\frac{1}{n} \sum_{j}^{n} f_{j h t}=F_{h t} .
$$

These steps produce $2 \times 4$ aggregate probabilistic forecast series and again $2 \times 4$ aggregate point forecast series (for GNP and IPD, and $h=1, \ldots, 4$, in each case).

Probabilistic and Point Forecasts: Mean Errors

Figure 3 compares each of the eight pairs of the $\Phi_{h}$ and $F_{h}$ series in terms of the discrepancies of the forecasts from common actual values, that is, the group mean errors $\left(\Phi_{h t}-A_{t}\right)$ and $\left(F_{h t}-A_{t}\right)$. It demonstrates that the paired mean error series are remarkably close in virtually all instances. 8

This is a strong finding of considerable significance. Evidently, the respondents on the whole equated their preferred (point) forecasts to the expected values or means of their predictive probability distributions. As shown below, the $\mathrm{pb}$ distributions tend to approximate symmetry. For unbiased forecasts, these results seem mildly suggestive of symmetrical loss functions, though they are not inconsistent with bias or asymmetrical loss functions for many of the individuals covered.

Actually, the forecasts clearly are not free of bias. Of the 46 mean errors of $\Phi$ predictions for GNP, H1-H4, (where $H$ denotes the horizon), 34 (74 percent) are negative. For IPD, the proportion of underestimates is still

$$
{ }^{8} \text { Note that }\left(\Phi_{h t}-A_{t}\right)-\left(F_{h t}-A_{t}\right)=\Phi_{h t}-F_{h t} \text {. Also, for any group of }
$$
respondents indexed $j=1, \ldots, n, \frac{1}{n} \sum_{j}^{n}\left(\phi_{j}-A\right)=\Phi-A$ and $\frac{1}{n} \sum_{j}^{n}\left(f_{j}-A\right)$ $=F-A$ (this applies to any $h$ and $t$, so these subscripts are omitted for simplicity). 

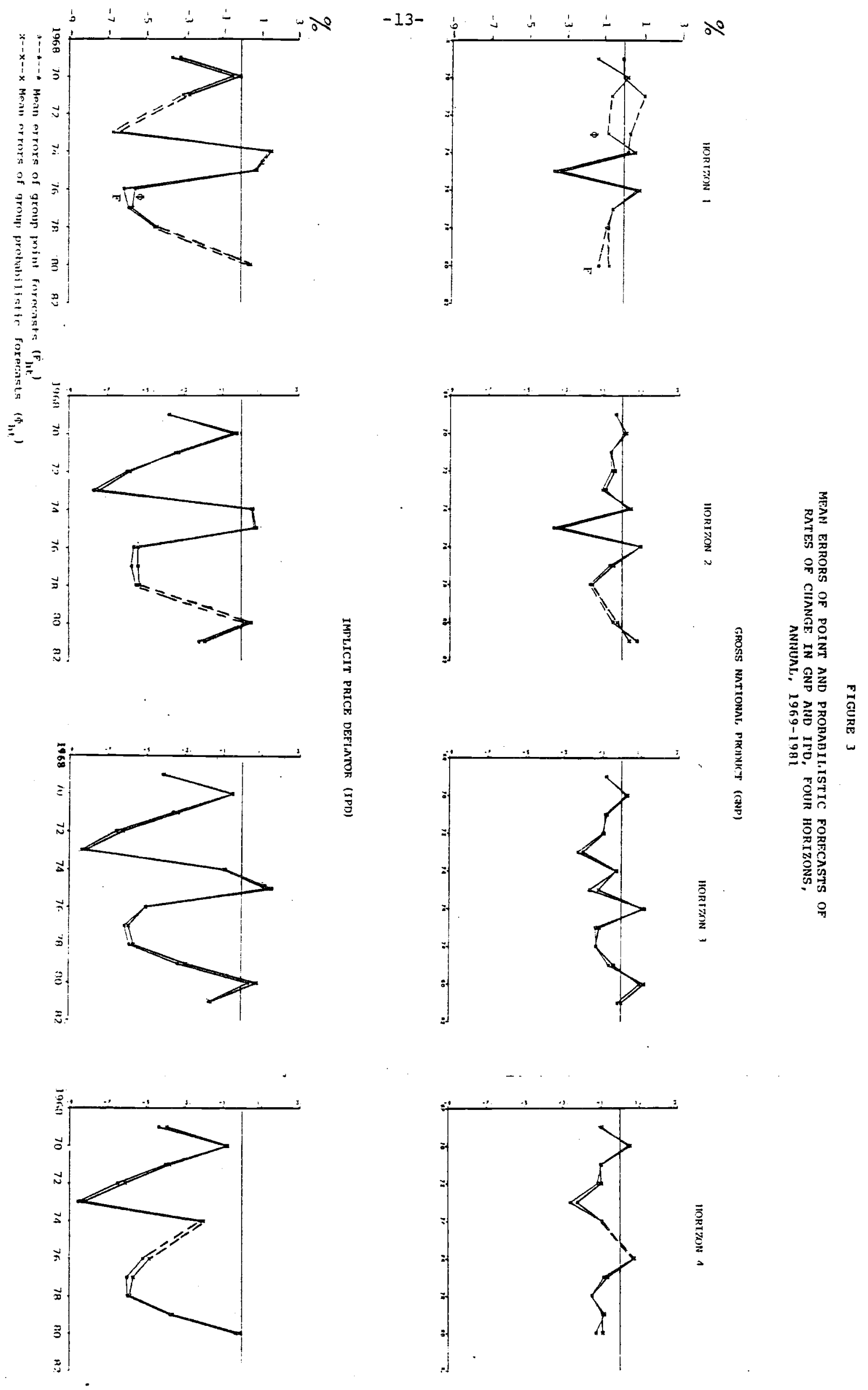
larger (38/46 or 838$)$. The underestimates are relatively large, particularly for IPD, while the overestimates are small. on the average, the mean errors are all negative, for either variable and each horizon, as shown in Table 1 , lines 1-4. Taken without regard to sign, the average error levels tend to increase with the horizon, while the corresponaing variability measures (in brackets) are rather remarkably stable. All of this refers to both the point forecasts (columns 2 and 4 ) and the mean probability forecasts (columns 3 and 5), confirming the close similarity of the paired $\Phi_{h}$ and $F_{h}$ series. $^{9}$ Series of Dispersion Measures

Figure 4 shows the series of the means of the standard deviations calculated from the individual probability forecast distributions ( $\bar{s}_{\phi}$ ) and matches them with the series of standard deviations for the corresponding sets of point forecasts $\left(s_{f}\right)$. For the GNP growth rates, $\bar{s}_{\phi}>s_{f}$ in each year at $H 1$ and in all but two years at $\mathrm{H} 2$, but the differences between $\bar{s}_{\phi}$ and $s_{f}$ are much smaller and less systematic at $H 3$ and $H 4$. For the IPD inflation rates, $\bar{s}_{\phi}$ exceed $s_{f}$ as a general rule (with exceptions of one year each at $H 1$ and $H 2$ and two years each at $H 3$ and $H 4)$. The $\bar{s}_{\phi}$ series vary much less over time than their $s_{f}$ counterparts.

Table 1 quantifies some of the inferences from these graphs (lines 5-8). The average levels of the $\bar{s}_{\phi}$ series vary narrowly from .80 to .98 for GNP and from .76 to .90 for IPD, with some propensity to increase between H1 and H3.

${ }^{9}$ The point forecasts for 1968-80 tend to underestimate the inflation (IPD) rates, increasingly so for the successive quarters of the year ahead, but they also overestimate the real growth (RGNP) rates, so that the changes in nominal GNP are predicted with little bias (zarnowitz, 1983a). However, the mean errors of the GNP forecasts for the successive quarters $(20, \ldots, 24)$, while small, are generally negative, and they cumulate. This produces much larger underestimation errors in the annual forecasts. Thus the average of the entries in lines $1-4$ of Table 1 , column 2 , is -.63 ; in contrast, the approximately comparable figure for the quarterly pt forecasts is -.17. 

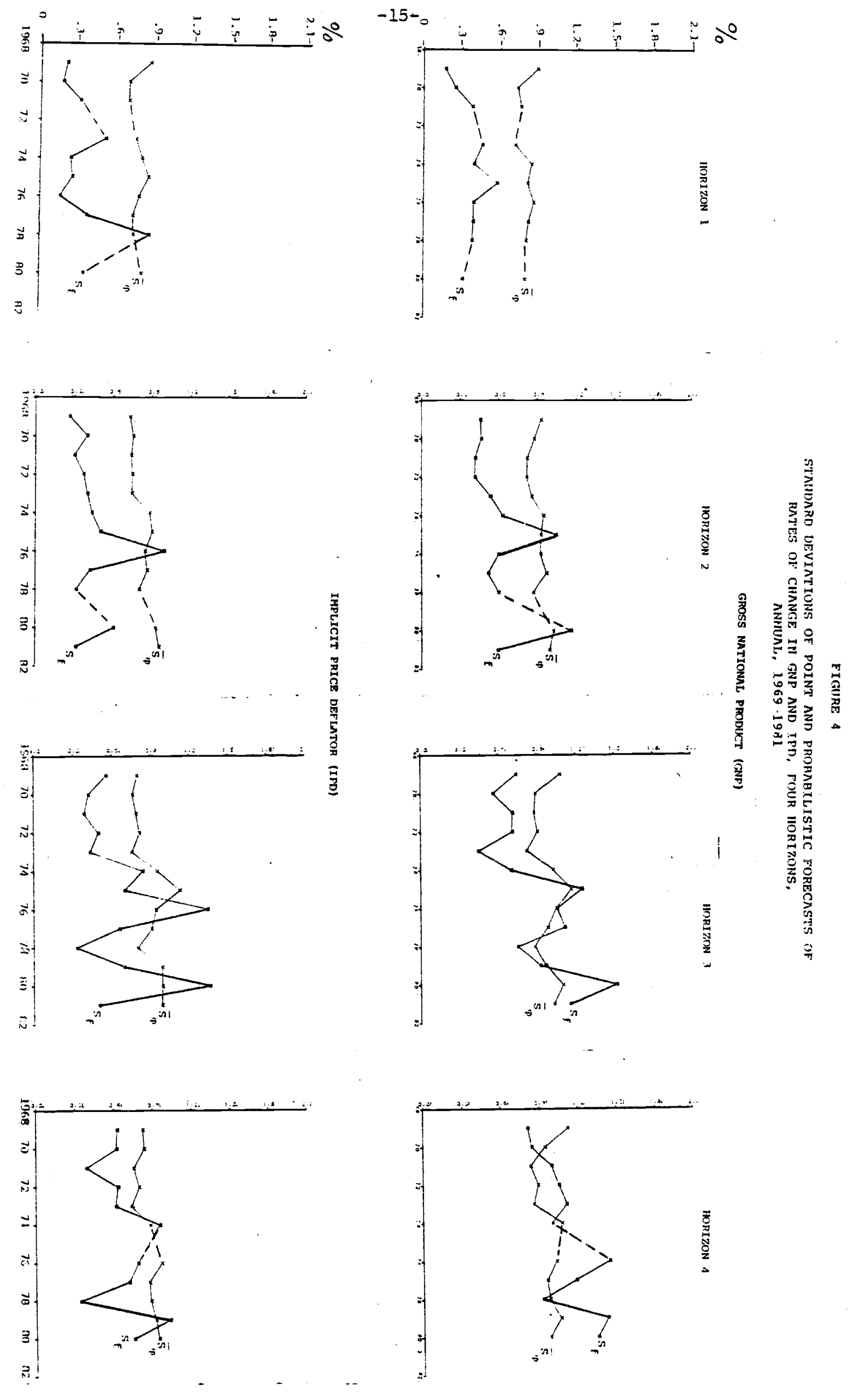


\section{Table 1}

Summary Statistics for Point Forecasts and Mean Probability Forecasts of Annual Percentage Changes in GNP and IPD, 1969-1981

Line Horizon

(1)

Gross National Product (GNP)
Point Mean Probability
Forecasts $\quad$ Forecasts

(2)
(3)

Mean Errors

Implicit Price Deflator (IPD)
Point Mean Probability
Forecasts Forecasts

(4)

(5)

$\begin{array}{lllllllll}1 & 1 & -.35 & (1.37) & -.60 & (1.42) & -2.43 & (2.98) & -2.45(3.03) \\ 2 & 2 & -.57 & (1.32) & -.55 & (1.32) & -3.49 & (2.99) & -3.36(2.88) \\ 3 & 3 & -.66 & (1.26) & -.65 & (1.32) & -3.69 & (3.02) & -3.51(2.92) \\ 4 & 4 & -.92 & (1.33) & -.98 & (1.38) & -4.56 & (2.48) & -4.27(2.45)\end{array}$

\section{Standard Deviations}

$\begin{array}{llllllllll}5 & 1 & .37 & (.11) & .80 & (.05) & .34 & (.21) & .76(.06) \\ 6 & 2 & .62 & (.24) & .91 & (.07) & .44 & (.20) & .83(.08) \\ 7 & 3 & .90 & (.31) & .98 & (.10) & .67 & (.34) & .90(.12) \\ 8 & 4 & 1.11 & (.22) & .98 & (.09) & .68(.21) & .86(.08)\end{array}$

NOTE :

For coverage, see the tabulation in text. Horizons 1, 2, 3, and 4 refer to forecasts of percentage change from year $t-1$ to year $t$ in the third, second, and first quarters of year $t$ and the fourth quarter of year $t$ - 1 , respectively. For both the mean errors (1ines 1-4) and the standard deviations of errors (lines 5-8), entries without brackets are means, entries within brackets the corresponding standard deviations. All measures are in percentage points, referring to the percent changes at annual rates. The entries in lines 1-4 correspond to the series shown in Figure 3, those in 1ines 5-8 to the series shown in Figure 4 . 
In contrast, the levels of the $s_{f}$ series are much lower at $H 1$ and increase strongly and monotonically with the horizon, from .37 to 1.11 for GNP and from .34 to .68 for IPD. The differences $\bar{s}_{\phi}-s_{f}$ are positive and relatively large in six of the eight categories (for GNP-H3 the difference is small, for GNP-H4 it is negative). In each case, the variability of the $\overline{\mathbf{s}}_{\phi}$ is much less than that of the paired $s_{f}$ series, as shown by the bracketed figures. Indeed, assuming our findings can be generalized, they have the important implication that the consensus statistics tend to understate uncertainty. As noted early in this paper, the use of common data, methods, and models would tend to narrow the differences even among the independently derived predictions of the widely monitored economic variables. The spread of influential views and interaction among experts and agents works in the same direction, producing further homogenization of the reported point forecasts.

One could argue that it is precisely when high uncertainty prevails that people will invest more resources in interactive prediction, with the result that their point forecasts or expectations would then tend to be more closely bunched. Also, in aiming at common targets in the not so near future about which little can be known or confidently prejudged, many individuals may try to avoid getting out on a limb; they would rather stay close to others and share in common errors than risk making eccentric errors. Under such conditions, strong consensus would be associated with high uncertainty (as in Figure 2, panel b).

If the above arguments had general validity, $s_{f}$ and $\bar{s}_{\phi}$ should be negatively correlated. ${ }^{10}$ However, it is easy to think of other hypotheses which would produce different results. Thus, if greater uncertainty induced ${ }^{10}{ }_{\text {Recall that the higher }} s_{f}$ denotes less consensus, while higher $\bar{s}_{\phi}$ is
interpreted as symptomatic of more uncertainty. 
greater interpersonal differentiation of expectations, the correlation between $s_{f}$ and $\bar{s}_{\phi}$ (called $r_{s}$ ) would be positive.

\begin{tabular}{|c|c|c|c|c|}
\hline \multirow[b]{3}{*}{ GNP } & \multicolumn{3}{|c|}{ Correlation Coefficients $\left(r_{s}\right)^{11}$ for } & \multirow[b]{2}{*}{$\mathrm{H} 4$} \\
\hline & $\mathrm{H} 1$ & $\mathrm{H} 2$ & H3 & \\
\hline & $-.23(.52)$ & $.61(.03)$ & $.76(.002)$ & $.09(.80)$ \\
\hline PD & $-.29(.42)$ & $.33(.29)$ & $.55(.05)$ & $.54(.09)$ \\
\hline
\end{tabular}

As the above tabulation shows, the findings on this score are mixed: of the eight $r_{s}$ coefficients, four are positive and significant at the ten percent level and others (of which two are positive and two negative) are not significantly different from zero. This is consistent with the notion that the different hypothetical situations we have considered, being by no means mutually exclusive, may all apply to a varying degree, frequently with mutually offsetting effects (although the prevalence of positive correlations deserves to be noted). This much is surely not implausible, although it is inevitably somewhat speculative: it must be emphasized again that our results are based on small samples (10-13 annual observations for each horizon) and cannot claim to be conclusive.

\section{Skewness and Kurtosis}

Figure 5 presents the pairs of skewness series for each horizon: an average of the measures for the individual probability distributions $\left(\overline{s k}_{\phi}\right)$ and statistics relating to the point forecasts from the same sources $\left(s k_{f}\right)$. The skewness coefficient is defined as

$$
s k=\frac{\mu_{3}}{\mu_{2}^{3 / 2}} \text {, }
$$

${ }^{11}$ The figures in parentheses are the associated probabilities that the correlations are due to chance. 
$-19$.
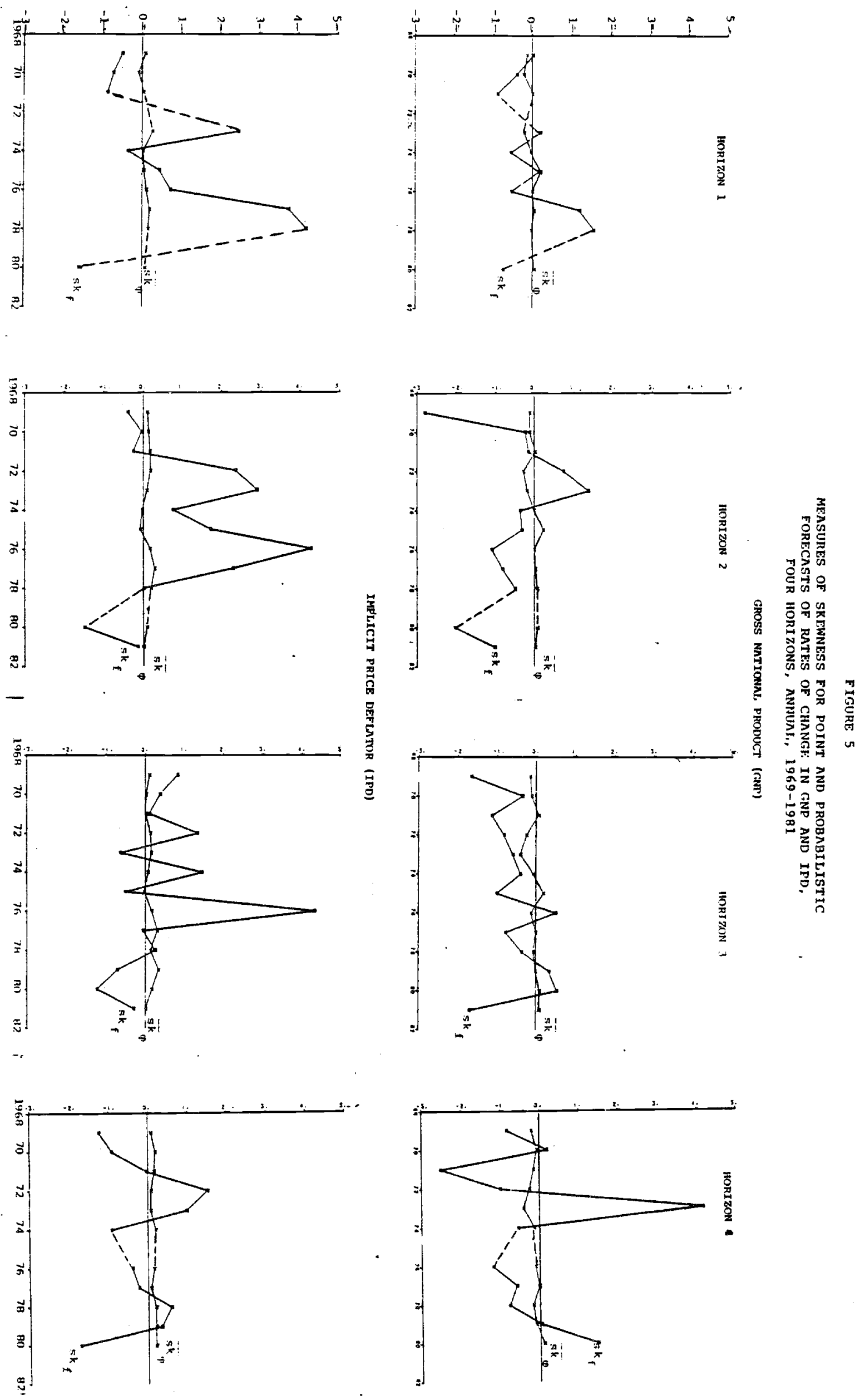
in terms of the calculated third and second moments about the mean. If the distribution is symmetrical, sk and $\mu_{3}$ equal zero; if it is not, sk will show the sign of the departure from symmetry and give some indication of its extent. 12

It is evident from the chart that, on the average, there is very little skewness in the probabilistic forecast distributions. Each of the $\overline{s k}_{\phi}$ series runs close to zero; the deviations are predominantly negative for the GNP growth and positive for the inflation predictions but they are small throughout, irrespective of the horizon. In contrast, the $s k_{f}$ series for the point forecasts fluctuate irregularly over broad ranges including numbers of both signs, although here, again, minuses prevail for GNP and pluses for IPD. No systematic horizon effects are discernible.

Figure 6 compares the series of kurtosis measures $k_{\phi}$ and $k_{f}$. The $k$ coefficient is calculated as a ratio of the fourth moment about the mean to the variance squared,

$$
k=\frac{\mu_{4}}{\mu_{2}^{2}} .
$$

This has the value 3 if the distribution is Normal. 12 on the average, the measures based on the individual probabilistic forecast distributions display no significant departures from that value: each of the $\overline{\mathrm{k}}_{\phi}$ series in the chart lies extremely close to the horizonal line drawn at the level of 3.00 . on the other hand, some of the $k_{f}$ series, particularly those for the inflation series, show great volatility, often attaining values much higher than 3 . This suggests that the underlying distributions of point forecasts across the

\footnotetext{
${ }^{12}$ See Kendall and Stuart, 1969, vol, 1, 85-86.
} 

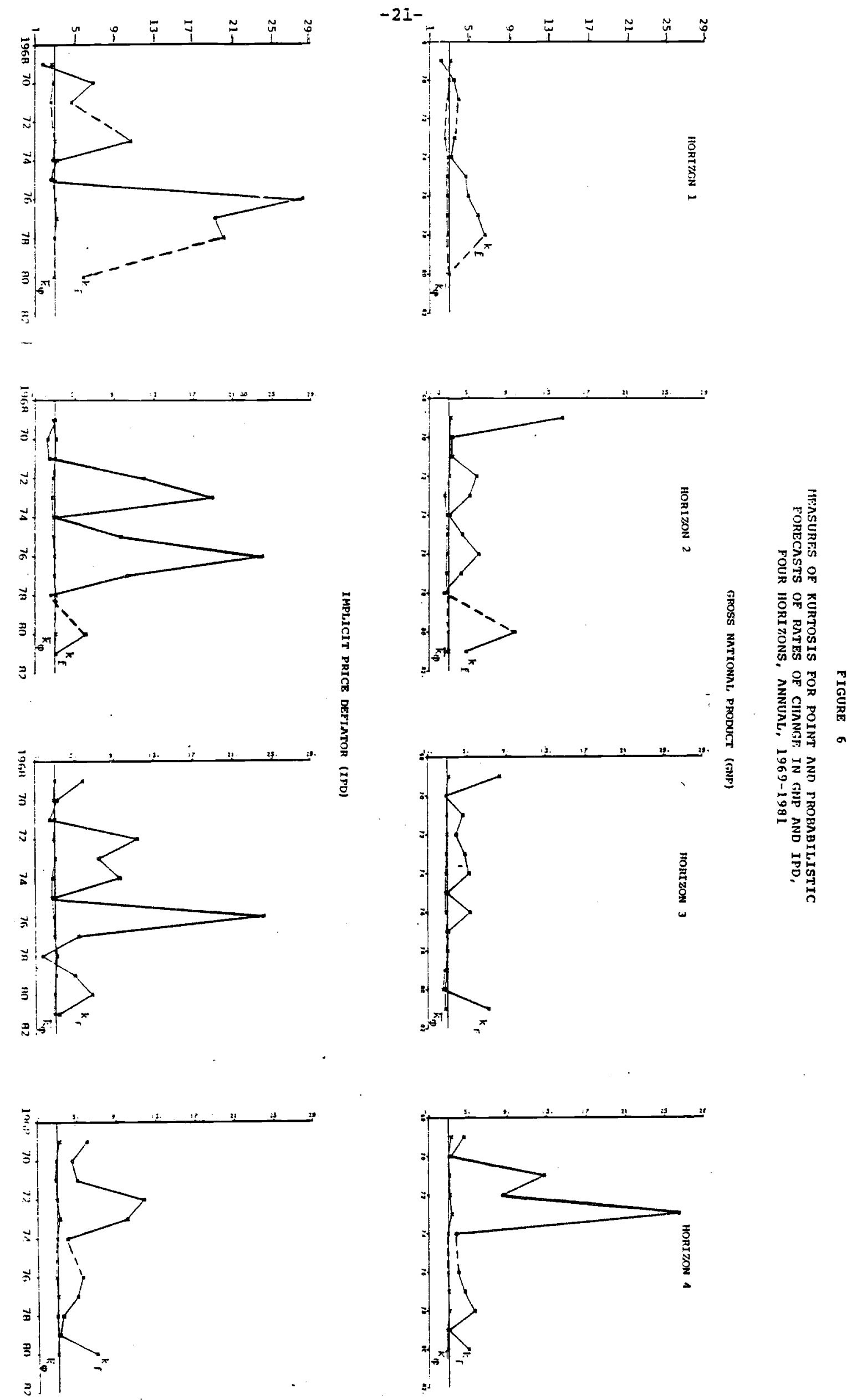
individuals may be leptokurtic, i.e., having sharper peaks and fatter tails than the Normal curve. ${ }^{13}$

III. Summary and Conclusions

This study starts from an operational distinction between "consensus" and "uncertainty." The former is defined as the degree of agreement among the corresponding point predictions reported by different forecasters; the latter refers to the diffuseness of the distributions of probabilities that the forecasters attach to the different possible values of an economic variable. Using this conceptual framework, section II addresses data on point and probabilistic forecasts of annual percentage changes in current-dollar GNP and the implicit price deflator (IPD). The source, here and later, is the quarterly ASA-NBER survey of macroeconomic forecasts, 1968-1981.

The matched mean point forecasts and mean probability forecasts agree closely. Apparently, on the whole, the prefered predictions coincide with the expected values of the probability distributions assessed by the survey respondents.

Measures of consensus such as the standard deviations of the point predictions, have been used as proxies for prior forecast uncertainty. Our own comparisons of the standard deviation of point forecasts and the mean standard deviation of predictive probability distributions indicate that the former tend to understate uncertainty as measured by the latter. This is so particularly for short horizons. There is some evidence that these measures of consensus and uncertainty are for the most part positively correlated.

${ }^{13}$ Symmetric leptokurtic distributions have been viewed as implying a considerable degree of consensus because of their peakedness; but they also have more outliers. See Schnader and Stekler, 1979. 
The distributions of point predictions in each period are, on the average, approximately symmetrical but they show considerable variation over time. These distributions tend to be leptokurtic, that is, they have heavier concentrations in the tails than in Normal distributions.

The examined predictive probability distributions are almost exclusively unimodal, with average measures of skewness and kurtosis conforming closely to Normal. The average standard deviations of the distributions increase only slightly with the forecast horizon (HO, ..., H4). 


\section{References}

Bates, J. M. and C. W. J. Granger (1969). "The Combination of Forecasts," Operations Research Quarterly 20: 451-468.

Cole, Rosanne, 1969. Errors in Provisional Estimates of Gross National Product. New York: National Bureau of Economic Researsh.

Cukierman, Alex, and Paul Wachtel. 1979. "Differential Inflationary Expectations and the Variability of the Rate of Inflation," American Economic Review 69 (September): 595-609.

Einhorn, Hillel, and Hogarth, Robin. 1975. "Unit Weighting Schemes for Decision Making," Organizational Behavior and Human Performance 13, no. 2 (April): 171-192.

Hogarth, Robin M., 1978. "A Note on Aggregating Opinions," Organizational Behavior and Human Performance 21: 40-46.

Kendall, Mauxice G., and Alan Stuart. (1975). The Advanced Theory of Statistics, 3rd edition. New York: Haffner.

Levi, M. D. and J. H. Makin, 1979. "Inflation Uncertainty and the Phillips Curve: Some Empirical Evidence," American Economic Review 70 (December): $1022-1027$.

Makin, John H. 1982. "Anticipated Money, Inflation Uncertainty and Real Economic Acitivity, Review of Economics and Statistics 64 , no. 1 (February): 126-134.

McNees, Stephen K., 1973. "The Predictive Accuracy of Econometric Forecasts," New England Economic Review (September/October): 3-27. Morris, Peter A. 1977. "Combining Expert Judgments: A Bayesian Approach," Management Science 23 (March): 679-693.

Mullineaux, Donald J., 1980. "Unemployment, Industrial Production and Inflation Uncertainty in the United States," Review of Economics and Statistics 62 (May): 163-169. 
Newbold, P. and C.W.J. Granger. 1974. "Experience with Forecasting Univariate Time Series and the Combination of Forecasts," Journal of the Royal Statistical Society A 137: 131-146.

Schnader, M.H., and H.O. Stekler. 1979. "The Existence and Evaluation of Economic 'Consensus' Forecasts," 1979 Proceedings of the Business and Economic Statistics Section, American Statistical Association, Washington, D.C.: $512-517$.

Zarnowitz, Victor. 1967. An Appraisal of Short-Term Economic Forecasts. New York: National Bureau of Economic Research. Zarnowitz, Victor. 1979. "An Analysis of Annual and Multiperiod Quarterly Forecsts of Aggregate Income, Output, and the Price Level," Journal of Business $\underline{52}$, no. 1 (January): 1-33. zarnowitz, Victor. 1982a. "On Functions, quality, and Timeliness of Economic Information," Journal of Business 55, no. 1 (January): 87-119. Zarnowitz, Victor. 1982b. "The Accuracy of Individual and Group Forecasts from Business Outlook Surveys," NBER Working Paper no. 1053 (December). zarnowitz, Victor. 1983. "Rational Expectations and Macroeconomic Forecasts," NBER Working Paper no. 1070 (January). 